\title{
Clinical efficacy of nab-paclitaxel in patients with metastatic pancreatic cancer
}

This article was published in the following Dove Press journal:

Drug Design, Development and Therapy

\section{Rossella De Luca \\ Livio Blasi² \\ Massimiliano Alù ${ }^{2}$ \\ Valerio Gristina' \\ Giuseppe Cicero'}

'Department of Surgical, Oncological and Oral Sciences, Section of Medical Oncology, University of Palermo, Palermo, Italy; ${ }^{2}$ Medical Oncology Unit, ARNAS Hospital Civico,

Di Cristina, Benfratelli, Palermo, Italy
Correspondence: Giuseppe Cicero Department of Surgical, Oncological and Oral Sciences, Section of Medical Oncology, University of Palermo, Via del Vespro, no 129, 90127 Palermo, Italy $\mathrm{Tel}+39916554531$

Fax +39916552549

Email giuseppe.cicero@unipa.it
Purpose: Pancreatic carcinoma is the neoplasia with the major mortality, and main standard treatments in this cancer increase survival but do not lead to complete recovery of the patient. The aim of this study was to evaluate the efficacy of Abraxane ${ }^{\circledR}$ (nab-paclitaxel) in Italian patients with metastatic pancreatic cancer (MPC).

Patients and methods: We conducted a retrospective analysis of 80 patients. Overall survival (OS) was the primary end point for evaluating the efficacy of nab-paclitaxel in combination with gemcitabine treatment, while carbohydrate antigen 19-9 (CA 19-9) reduction, safety, progression-free survival (PFS), overall response rate and reduction in pain were secondary end points.

Results: The median OS was 8 months, and the median PFS was 5 months. A considerable difference in CA 19-9 before and after treatment was observed. Descriptive and correlation analyses were done to examine the relationship between CA 19-9 response and OS. Linear regression analysis between OS and CA 19-9 response revealed that CA 19-9 is an important predictor of OS, showing a positive correlation.

Conclusion: Nab-paclitaxel is a well-tolerated and effective treatment for patients affected by MPC. The drug showed an improved tolerability profile, significant pain relief and an increase in survival rate.

Keywords: Abraxane, chemotherapy, pancreatic carcinoma, metastasis, CA 19-9, pain, overall survival

\section{Introduction}

Pancreatic cancer (PA) is the fifth leading cause of death in Europe for cancer-related reasons, with $80 \%$ of patients ineligible for potential curative resection at diagnosis. In these cases, PA is diagnosed in a locally advanced stage or is metastatic with a particularly poor prognosis ${ }^{1}$ and a total survival rate $6 \%$ at 5 years. ${ }^{2,3}$ Treatment with the long-term standard care, gemcitabine (Gem) monotherapy, led to a median overall survival (OS) of $\sim 5-7$ months in multiple Phase III trials of patients with metastatic pancreatic cancer (MPC). ${ }^{4}$ Gemcitabine in association with oxaliplatin (GEMOX) has demonstrated discrete efficacy in metastatic or locally advanced cancer with a median duration of 4.5 months response with a significant neurological toxicity due to oxaliplatin. ${ }^{5}$ In 2011, Conroy et $\mathrm{al}^{6}$ published the results of a Phase II/III study that demonstrated greater efficacy of Gem in MPC patients, highlighting a clinically meaningful survival benefit and better quality of life for a regimen composed of 5-fluorouracil, folinic acid (leucovorin), irinotecan and oxaliplatin (FOLFIRINOX) at the expense of increased toxicity. Recent combinations of chemotherapy and target therapy such as erlotinib plus Gem have achieved a significant, albeit modest, increase in OS vs Gem in a global study of locally advanced or MPC patients. ${ }^{7}$ The phase III trial, Metastatic 
Pancreatic Adenocarcinoma Clinical Trial (MPACT), showed improved efficacy of Abraxane ${ }^{\circledR}$ (nab-paclitaxel; Celgene, Boudry, Switzerland) plus Gem compared to Gem alone. An updated OS analysis showed a 2-month difference in the arm with nab-paclitaxel plus Gem vs Gem alone (median, 8.7 vs 6.6 months) and a total reduction in the risk of death of $28 \%$, an improvement in survival at 1 year of $59 \%$ and an improvement in survival rates at 2 years of $12 \%$. A statistically significant improvement in progression-free survival (PFS) was noted for patients treated with nab-paclitaxel plus Gem compared to Gem alone, with a 1.8-month increase in PFS and overall response rate (ORR) ${ }^{8,9} \mathrm{Nab}$-paclitaxel is a targeted chemotherapy with a targeted and more selective action that uses "nab" technology, nanotechnology. This technology uses albumin, a naturally occurring human protein almost in nanometric size, containing chemotherapeutic drugs that are then transported directly to the tumor site. ${ }^{10}$ According to the studies in the literature, the main purpose of this retrospective study was to evaluate in our clinical reality the safety and efficacy of nab-paclitaxel in patients with MPC. In particular, OS analysis was evaluated as the primary end point. Secondary end points were carbohydrate antigen 19-9 (CA 19-9) reduction, pain relief, tolerability and PFS. ${ }^{11,12}$

\section{Study design}

This retrospective study was conducted to evaluate the safety and efficacy of nab-paclitaxel in association with Gem in the first-line treatment of adult patients with metastatic pancreatic adenocarcinoma. We retrospectively analyzed 80 patients with metastatic pancreatic carcinoma between June 2015 and June 2017 at the Medical Oncology Unit of the University Palermo and ARNAS Hospital Civico of Palermo. The study was approved by the ethics committee of Policlinico "P Giaccone", Palermo, and all patients provided written informed consent to review their medical records. The nabpaclitaxel was administered up to progression of the disease (PD) or the development of an unacceptable toxicity.

\section{Patients and methods}

\section{Patients' selection}

We considered only patients who met the following inclusion criteria: 1) histologically or cytologically confirmed diagnosis of metastatic pancreatic adenocarcinoma; 2) performance status 0-2 according to the Eastern Cooperative Oncology Group (ECOG); 3) clinical and radiological evidence of metastatic disease (number of lesion $\geq 1$ according to the Response Evaluation Criteria in Solid Tumors (RECIST; v 1.1); and 4) adequate liver, renal and blood functionality (neutrophils
$>2.0 \times 10^{9} / \mathrm{L}$, platelets $>100 \times 10^{9} / \mathrm{L}$, hemoglobin $>10 \mathrm{~g} / \mathrm{dL}$, creatinine $<1 \mathrm{mg} / \mathrm{dL} \times$ upper limit of normal [ULN], bilirubin $<1 \times$ ULN, AST and ALT $<5 \times$ ULN). Similar to previous studies, ${ }^{13-15}$ we excluded patients who showed a hypersensitivity to nab-paclitaxel and its excipients or other formulation components; diagnosis of other malignancies, with the exception of skin basal cell carcinoma and serious comorbidities not adequately checked by other ongoing therapies (eg, liver disease, diabetes, infections, heart disease, etc.).

\section{Study assessments}

\section{Evaluation of the response rate and toxicity}

The response rate in terms of reduction in CA 19-9 and measurable pathology (at 8 weeks after the end of treatment) was evaluated before the beginning of each treatment cycle and then every 3 months until progression of the disease. The spiral CT scan with and without contrast medium was always done before the start of each treatment cycle, then every 3 months until disease progression and whenever an increase in CA 19-9 or a presumed clinical progression was reported. Total-body PET was done if advised by the oncologist for any suspicion of disease progression. For those patients who did not show a measurable pathology, a sustained reduction of $\geq 50 \%$ in serum levels of CA 19-9 was observed as treatment response to baseline values. Progression of CA 19-9 was considered as 1 ) an increase in CA $19-9 \geq 25 \%$ compared to baseline values in those patients who did not achieve a significant reduction $(\geq 50 \%)$ in serum levels of CA 19-9 during treatment, and 2) a 50\% increase in the lowest observed level in patients who achieved a significant reduction $(\geq 50 \%)$ in serum levels of CA 19-9 during treatment. The toxicity associated with the treatment and the side effects were evaluated after each course of treatment and reported according to the Cancer Therapy Evaluation Program, Common Terminology Criteria for Adverse Events (CTCAE), version 4.0.

\section{Modality of administration}

The recommended dose of nab-paclitaxel was $125 \mathrm{mg} / \mathrm{m}^{2}$, administered intravenously within 30 minutes on days 1,8 and 15 of each 28-day cycle. The recommended concomitant dose of Gem was $1,000 \mathrm{mg} / \mathrm{m}^{2}$ administered intravenously within 30 minutes immediately after completion of nabpaclitaxel administration on days 1, 8 and 15 of each 28-day cycle. Patients were premedicated at least 30 minutes before the infusion with steroid (sodium dexamethasone phosphate), antiemetics, antihistamines and $\mathrm{H} 2$ antagonists. In addition, during the entire treatment period, the patient was advised to have adequate hydration to prevent complications such as 
renal failure. The treatment was administered up to the PD or the development of an unacceptable toxicity. According to the clinical practice procedures, the therapy was postponed for up to 2 weeks if the neutrophil count was $<1.5 \times 10^{9} / \mathrm{L}$, platelet count was $<100 \times 10^{9} / \mathrm{L}$, hemoglobin level was $<8.5 \mathrm{~g} / \mathrm{dL}$ or bilirubin and/or transaminase levels were $>1.5 \times \mathrm{ULN}$. In the case of neutropenia (G2-G3), G-CSF was also given beforehand, subcutaneously. If major anemia of neutropenia (G2-G3), blood transfusions are done or in less severe cases, erythropoietin vial subcutaneously, and finally for thrombocytopenia the steroid therapy or the platelet endovenous infusion.

\section{Statistical analyses}

The normality of the distribution was checked using the univariate indices of skewness and kurtosis with an acceptance threshold equal to 1 . All variables complied with normality indices. Descriptive statistics were useful to provide a sociodemographic representation and the distribution of all variables. The disease control rate was defined as the percentage of patients with an objective response and/or stable disease (SD) lasting $>5$ months. Inferential statistical analyses were performed to detect any significant relationships among the considered variables. PFS and OS were calculated from the start date of the treatment until the date of disease progression or death from any cause for PFS and until the date of the last follow-up, death or the final follow-up day of the evaluation for OS. The follow-up date was June 2017. PFS and OS curves were estimated by using the KaplanMeier method. To calculate the sample size, a reduction in CA $19-9$ of at least $25 \%$ from baseline was considered to be clinically relevant, with an $\alpha$ error of $5 \%$ and a statistical power of $80 \%$, as reported in the literature. The relationship between OS and CA 19-9 was analyzed by linear regression (OS was the dependent variable $Y$, and the CA 19-9 response was the independent variable $X$ ). The goodness of fit was assessed with the coefficient of determination $R^{2}$, which represents the variability proportion between the observed values for $Y$, as explained by the linear regression of $Y$ over $X$. The slope values could be interpreted as an increase in $Y$ at every increase in $X$ values. Data were represented via a scatter plot and regression line. A $p$-value threshold of 0.05 was used to evaluate the significance of the obtained data. Statistical analysis was performed using the Statistical Package for the Social Sciences (SPSS) software, version 22.0.

\section{Results}

\section{Treatment exposure and follow-up}

Demographic, clinical and pathological features of 80 patients with MPC are reported in Table 1. At the time of diagnosis,
Table I Baseline demographic and clinical characteristics $(\mathrm{N}=80)$

\begin{tabular}{ll}
\hline Characteristics & Patients n (\%) \\
\hline Mean age (range), years & $62(48-75)$ \\
Sex & \\
$\quad$ Male & $42(52)$ \\
$\quad$ Female & $38(48)$ \\
ECOG performance status & \\
0 & $8(10)$ \\
I & $38(47.5)$ \\
2 & $34(42.5)$ \\
Median CA 19-9 level (range), ng/mL, <59 cutoff & $150(80-1,200)$ \\
Primary tumor location & \\
Head & $34(42)$ \\
Body & $23(28)$ \\
Tail & $8(10)$ \\
Metastatic site & \\
Liver & $58(72.5)$ \\
Lung & $19(24)$ \\
Peritoneum & $8(10)$ \\
Biliary stent & $22(27.5)$ \\
Previous Whipple procedure & $8(10)$ \\
\hline
\end{tabular}

Abbreviations: ECOG, Eastern Cooperative Oncology Group; CA 19-9, carbohydrate antigen 19-9.

patients were aged between 48 and 75 years, with a mean age of 62 years. The response rate of CA 19-9 in 8 weeks, after the end of treatment, was 55\%. Treatment with nab-paclitaxel in combination with Gem was well tolerated and gave a good level of disease control (partial response [PR] $+\mathrm{SD}, 55 \%$ ). After an average 6-month follow-up duration (range 3-8 months), 18 (22.5\%) patients had a PR to therapy, $26(32.5 \%)$ patients had an SD and $36(45 \%)$ patients had PD.

\section{CA $19-9$ reduction}

In our cohort of patients, we found a significant impact using nab-paclitaxel plus Gem on CA 19-9 reduction ( $>50 \%)$. In particular, the mean reduction was 55\% (95\% CI: $38.67-$ 54.63) and was consistent with data already published. This reduction was related to a better survival (Figure 1). Linear regression analysis between OS and CA 19-9 response revealed that CA 19-9 is an important predictor of OS, showing a positive correlation with $\operatorname{OS}(\beta=0.365, p<0.01)$. The explained variance, in this analysis, was $55 \%\left(R^{2}=0.60\right)$ with a slope of 1.46 ( $p=0.0032$; Figure 1). The Bravais-Pearson index showed a positive correlation between the response rate of CA 19-9 and OS, with a value of 0.60 (95\% CI: 0.25-0.87; $p=0.003$; Table 2).

\section{OS analysis}

Among 80 patients enrolled in this study, updated interim analysis of survival (until the last follow-up in June 2017) 


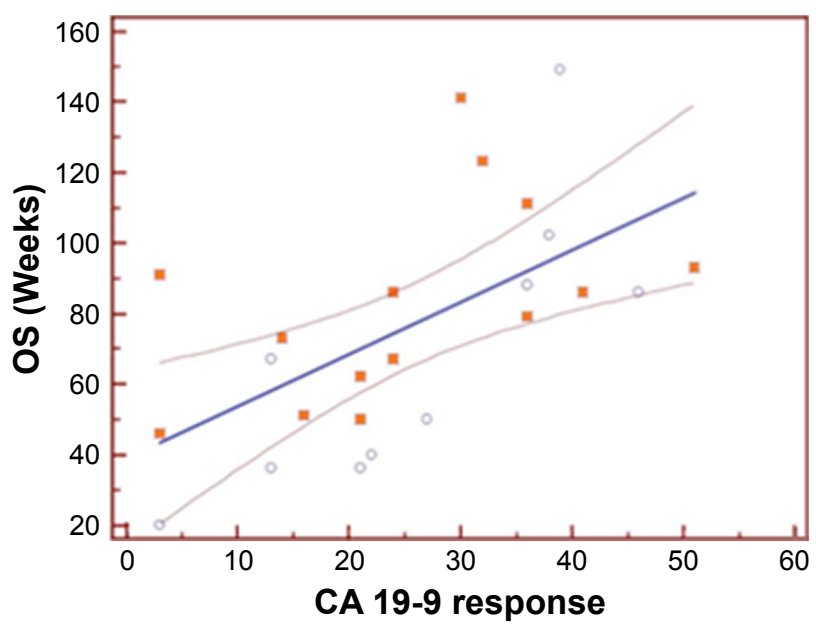

Figure I Regression analysis between CA I9-9 response and OS of patients $(\mathrm{N}=80)$. Abbreviations: CA 19-9, carbohydrate antigen 19-9; OS, overall survival.

showed a median OS of 8 months (95\% CI: 7.13-8.86; Figure 2).

\section{Time of PFS analysis}

In this study, median duration of response was 5 months (95\% CI: 3.86-6.13), while median time of progression in patients with PR or SD was 5.13 months (95\% CI: 4.13-6.14; Figure 3).

\section{Tolerability}

In the group of patients, nab-paclitaxel plus Gem treatment were well tolerated. Treatment-related toxicity was not significant, and the side effects were evaluated after each course of therapy and reported in accordance with the Cancer Therapy Evaluation Program, CTCAE, version 4.0. The most common adverse events were G1-G2 anemia that required the use of erythropoietin subcutaneously, G1-G2 neutropenia, a few G3 cases requiring the use of G-CSF, grades G1-G2 thrombocytopenia corrected with the use of corticosteroids and finally alopecia that occurred in $15 \%$ of patients. Events associated with the treatment of toxicity and side effects are described in Tables 3 and 4, respectively. No patient had treatment interrupted due to severe side effects, but adverse events of

Table 2 Pearson's correlation among CA 19-9 and OS in the treatment with Abraxane ${ }^{\circledR}$ (nab-paclitaxel; Celgene, Boudry, Switzerland; $N=80$ )

\begin{tabular}{lll}
\hline Variable & CA 19-9 & OS \\
\hline CA 19-9 & I & $0.60 * *$ \\
OS & $0.60 * *$ & 1 \\
\hline
\end{tabular}

Notes: ${ }^{* *} p<0.01$. Bold figures indicate the significance index of the relation between variables.

Abbreviations: CA 19-9, carbohydrate antigen 19-9; OS, overall survival.

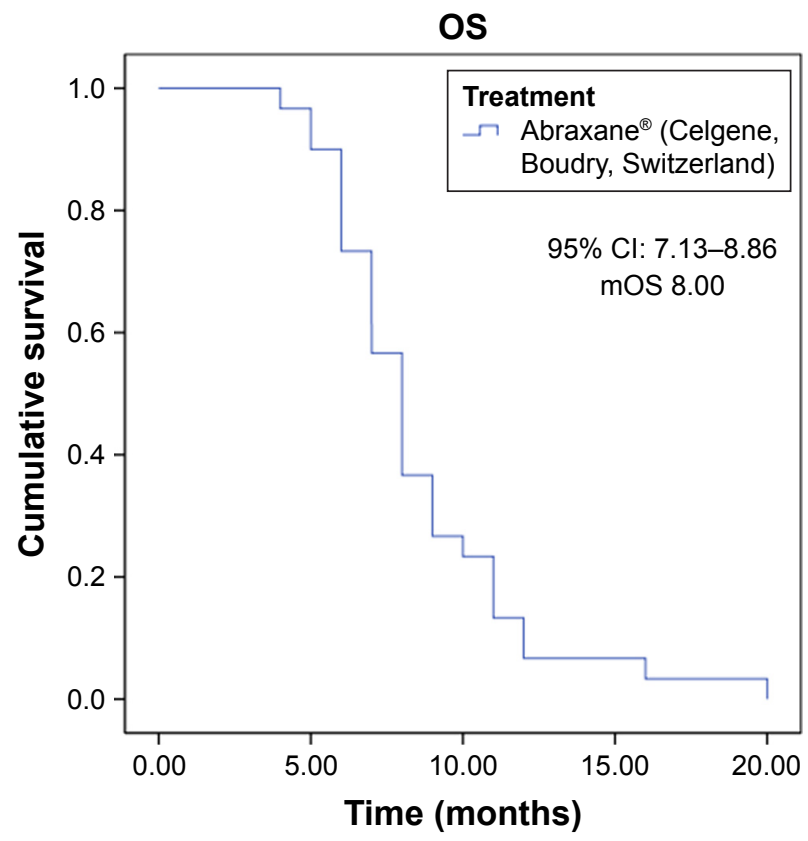

Figure 2 Kaplan-Meier plot of mOS (interim analysis; $\mathrm{N}=80$ ). Abbreviations: mOS, median overall survival; OS, overall survival.

grade $\geq 3$ were effectively treated with dose reductions and delays (Tables 3 and 4).

\section{Response to pain}

Pain reduction assessed by the visual analog scale (VAS) showed a response to pain in $34 \%$ of patients. In addition, improvement

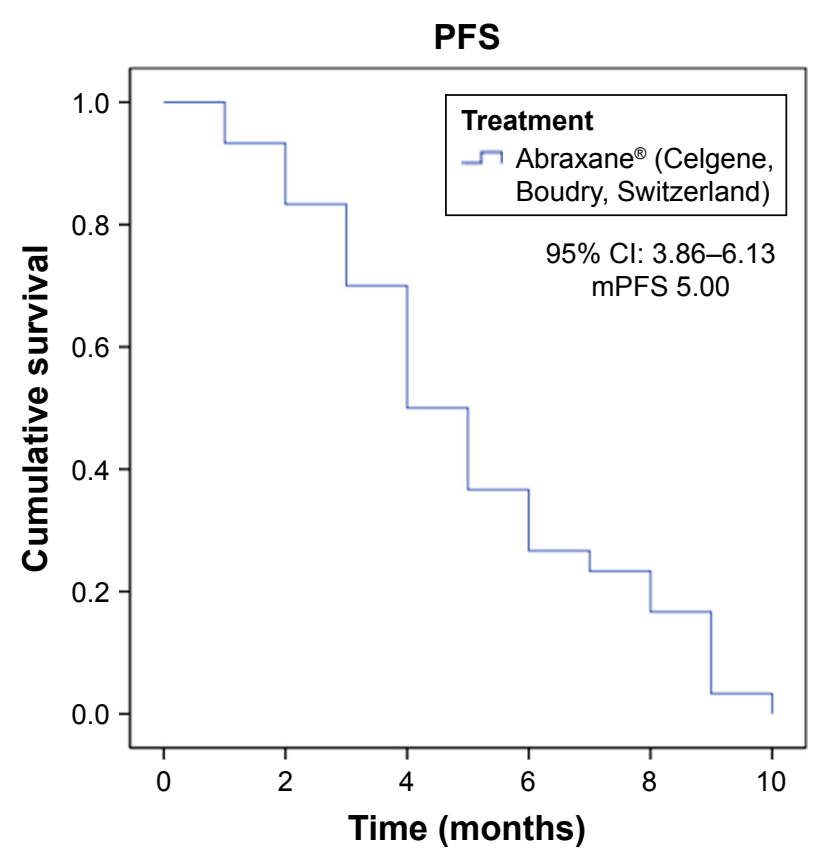

Figure 3 Kaplan-Meier plot of mPFS (interim analysis; $\mathrm{N}=80$ ).

Abbreviations: mPFS, median progression-free survival; PFS, progression-free survival. 
Table 3 Treatment-associated toxicity $(\mathrm{N}=80)$

\begin{tabular}{ll}
\hline Adverse events of any grade & No of patients (\%) \\
\hline Hematologic & $38(47.5)$ \\
Neurologic & $10(12.5)$ \\
Gastrointestinal & $21(26)$ \\
Infections & $6(7)$ \\
Metabolic & $13(16)$ \\
Flu-like symptoms & $7(8)$ \\
Cardiovascular & $2(2.5)$ \\
\hline
\end{tabular}

in quality of life was identified with a reduction in pain symptoms in $58.3 \%$ of patients with a VAS score of $<22$ points and a reduced use of analgesics in $48 \%$ of patients.

\section{Discussion}

In Western countries, $\mathrm{PA}$ is one of the most frequent cancers with a high mortality rate. This study aims to evaluate the efficacy of the combination of a new chemotherapy-targeted drug nab-paclitaxel in association with Gem in Italian patients with metastatic pancreatic adenocarcinoma, as the first line of treatment. ${ }^{16}$ Our findings are consistent with a recent and up-to-date analysis of the survival data of the study MPACT, a Phase III study comparing nab-paclitaxel plus Gem with monotherapy based on Gem alone. In the MPACT trial, nabpaclitaxel plus Gem demonstrated a longer OS (median, 8.5 vs 6.7 months; hazard ratio [HR], 0.72 ; 95\% CI: $0.62-0.83$; $p<0.001) .{ }^{17}$ Our analysis shows that treatment was well tolerated and was associated with an increase in OS and PFS with a good overall response rate (ORR) and a reduction in CA 19-9 levels. In particular, CA 19-9 reduction was $>50 \%$, and we also demonstrated that this reduction was linked to an increase in the OS. Therefore, the CA 19-9 response may be an important predictor of an increase in OS with a positive

Table 4 Adverse events graded according to CTCAE, version $4.0(\mathrm{~N}=80)$

\begin{tabular}{ll}
\hline Adverse event & No of patients (\%) \\
\hline Hematological & \\
Grades 2-3 anemia & $15(8)$ \\
Grades I-2 neutropenia & $23(28)$ \\
Grade 3 neutropenia & $11(14)$ \\
Grade 3 thrombocytopenia & $10(12.5)$ \\
Febrile neutropenia & $5(6)$ \\
Leukopenia & $10(12.5)$ \\
Non-hematological & \\
Grade 3 asthenia & $27(33.7)$ \\
Grades 2-3 peripheral neuropathy & $18(22.5)$ \\
Grade 3 stomatitis & $8(10)$ \\
Grade 2 alopecia & $13(15)$ \\
Grades 2-3 diarrhea & $9(11)$ \\
\hline
\end{tabular}

Abbreviation: CTCAE, Common Terminology Criteria for Adverse Events. correlation obtained between the CA 19-9 response and OS ( $\beta=0.365, p<0.01)$. However, our findings are in line with those reported in the literature, with an OS of 8 months $(95 \%$ CI: 7.13-8.86) and a PFS of 5 months (95\% CI: 3.86-6.13); this work has a limit that must be taken into account, for example, the small sample size. Nevertheless, we can state that in the first line of treatment of MPC, nab-paclitaxel plus Gem therapy is a valid therapeutic option that can be considered standard. The treatment shown an improvement in the quality of life with a reduction in pain. ${ }^{18,19}$ In addition, the treatment showed a good safety and tolerability profile; neutropenia was the most frequent side-effect associated with treatment, and only $14 \%$ of patients developed severe neutropenia (CTCAE grade 3) treated with G-CSF. This result is in accordance with published data (Studio MPACT) showing a greater risk of mild to moderate side effects associated with the treatment. ${ }^{9,20}$ In 2017, McBride et al compared patients treated with nab-paclitaxel plus Gem vs FOLFIRINOX. The authors demonstrated that the two treatments had a similar duration but lower costs of outpatient prescriptions, treatment administration and supportive care in nab-paclitaxel plus Gem. ${ }^{18}$ These data indicate that nab-paclitaxel plus Gem has a manageable safety profile and is an effective first-line option in patients in Western Europe with MPC. ${ }^{21-23}$

Nab-paclitaxel is a target therapy with nanotechnology, which allows transporting the drug at optimal concentrations directly to the tumor lesion; furthermore, nab-paclitaxel shows a good efficacy and safety profile. Considering other fluorobased (FOLFIRINOX) schema therapies, nab-paclitaxel showed minor toxicity and more safety in fragile and comorbid patients. ${ }^{24}$ Prospective studies and cohort studies have demonstrated that therapy with nab-paclitaxel, directly or indirectly compared with other Gem-based, platinum derivative and fluorine-based (FOLFIRINOX) therapies, has greater efficacy and lower toxicity in the setting of patients who frequently have peritoneal metastasis and biliary stand. ${ }^{25,26}$ Finally, use of nab-paclitaxel in the first line of treatment, as suggested in the guidelines, allows performance of a second line of treatment with other drugs. ${ }^{27}$

\section{Conclusion}

Our findings, even though with a limited number of patients, are consistent with clinical evidence reported in the literature. The combination of the two drugs nab-paclitaxel plus Gem was effective, well tolerated and safe in patients with metastatic pancreatic adenocarcinoma, and the toxicity associated with the treatment is acceptable. ${ }^{28,29}$ Additionally, 
more than half of the patients being treated with $P D$ received a subsequent therapy, indicating that nab-paclitaxel plus Gem was also a feasible first-line option on which it is possible to build a treatment plan. Pain response rates and quality of life are satisfactory. ${ }^{30}$ Based on these findings, we can conclude that treatment with nab-paclitaxel plus Gem is a valuable therapeutic regimen for the progressive form of MPC.

\section{Disclosure}

The authors report no conflicts of interest in this work.

\section{References}

1. Kamisawa T, Wood LD, Itoi T, Takaori K. Pancreatic cancer. Lancet. 2016;388(10039):73-85.

2. Siegel RL, Miller KD, Jemal A. Cancer statistics. CA Cancer J Clin. 2015;65:5-29.

3. Sohal DP, Mangu PB, Khorana AA, et al. Metastatic pancreatic cancer: American Society of Clinical Oncology Clinical Practice Guideline. J Clin Oncol. 2016;34(23):2784-2796.

4. Balaban EP, Mangu PB, Khorana AA, et al. Locally advanced, unresectable pancreatic cancer: American Society of Clinical Oncology Clinical Practice Guideline. J Clin Oncol. 2016;34(22):2654-2668.

5. Louvet C, Labianca R, Hammel P, et al; GERCOR, GISCAD. Gemcitabine in combination with oxaliplatin compared with gemcitabine alone in locally advanced or metastatic pancreatic cancer: results of a GERCOR and GISCAD phase III trial. J Clin Oncol. 2005;23(15): 3509-3516.

6. Conroy T, Desseigne F, Ychou M, et al; Groupe Tumeurs Digestives of Unicancer, PRODIGE Intergroup. FOLFIRINOX versus gemcitabine for metastatic pancreatic cancer. $N$ Engl J Med. 2011;364(19): $1817-1825$.

7. Mantripragada KC, Safran H. Optimizing initial chemotherapy for metastatic pancreatic cancer. Future Oncol. 2016;12(9):1125-1133.

8. Cunningham D, Chau I, Stocken DD, et al. Phase III randomized comparison of gemcitabine versus gemcitabine plus capecitabine in patients with advanced pancreatic cancer. J Clin Oncol. 2009;27(33): $5513-5518$.

9. Tabernero J, Chiorean EG, Infante JR, et al. Prognostic factors of survival in a randomized phase III trial (MPACT) of weekly nab-paclitaxel plus gemcitabine versus gemcitabine alone in patients with metastatic pancreatic cancer. Oncologist. 2015;20(2):143-150.

10. Cohen SJ, O'Neil BH, Berlin J, et al. A phase $1 \mathrm{~b}$ study of erlotinib in combination with gemcitabine and nab-paclitaxel in patients with previously untreated advanced pancreatic cancer: an Academic Oncology GI Cancer Consortium study. Cancer Chemother Pharmacol. 2016; 77(4):693-701.

11. Oettle H, Richards D, Ramanathan RK, et al. A phase III trial of pemetrexed plus gemcitabine versus gemcitabine in patients with unresectable or metastatic pancreatic cancer. Ann Oncol. 2005;16(10):1639-1645.

12. Vogel A, Römmler-Zehrer J, Li JS, McGovern D, Romano A, Stahl M. Efficacy and safety profile of nab-paclitaxel plus gemcitabine in patients with metastatic pancreatic cancer treated to disease progression: a subanalysis from a phase 3 trial (MPACT). BMC Cancer. 2016; 16(1):817.

13. Cicero G, De Luca R, Dieli F. Efficacy and safety of the oral multikinase Regorafenib in metastatic colorectal cancer. Oncology. 2017;93(6): 354-358.

14. Cicero G, De Luca R, Blasi L, et al. Treatment with abiraterone in metastatic castration-resistant prostate cancer patients progressing after docetaxel: a retrospective study. Anticancer Drugs. 2017;28(9): $1047-1052$.
15. De Luca R, Costa RP, Tripoli V, Murabito A, Cicero G. The clinical efficacy of radium-223 for bone metastasis in patients with castrationresistant prostate cancer: an Italian clinical experience. Oncology. 2017; 94(3):161-166.

16. Von Hoff DD, Ervin T, Arena FP, et al. Increased survival in pancreatic cancer with nab-paclitaxel plus gemcitabine. N Engl J Med. 2013; 369(18):1691-1703.

17. Chiorean EG, Von Hoff DD, Reni M, et al. CA19-9 decrease at 8 weeks as a predictor of overall survival in a randomized phase III trial (MPACT) of weekly nab-paclitaxel plus gemcitabine versus gemcitabine alone in patients with metastatic pancreatic cancer. Ann Oncol. 2016;27(4):654-656.

18. Picozzi V, Narayanan S, Henry Hu X, Vacirca J. Health-related quality of life in patients with metastatic pancreatic cancer. J Gastrointest Cancer. 2017;48(1):103-109.

19. Ottaiano A, Capozzi M, DE Divitiis C, et al. Nab-paclitaxel and gemcitabine in advanced pancreatic cancer: the one-year experience of the National Cancer Institute of Naples. Anticancer Res. 2017; 37(4):1975-1978.

20. Fountzilas C, Chhatrala R, Khushalani N, et al. A phase II trial of erlotinib monotherapy in advanced pancreatic cancer as a first- or second-line agent. Cancer Chemother Pharmacol. 2017;80(3):497-505.

21. McBride A, Bonafede M, Cai Q, et al. Comparison of treatment patterns and economic outcomes among metastatic pancreatic cancer patients initiated on nab-paclitaxel plus gemcitabine versus FOLFIRINOX. Expert Rev Clin Pharmacol. 2017;10(10):1153-1160.

22. Ottaiano A, Capozzi M, DE Divitiis C, et al. Nab-paclitaxel and gemcitabine in advanced pancreatic cancer: the one-year experience of the National Cancer Institute of Naples. Anticancer Res. 2017;37(4): 1975-1978.

23. Mato-Berciano A, Raimondi G, Maliandi MV, Alemany R, Montoliu L, Fillat C. A NOTCH-sensitive uPAR-regulated oncolytic adenovirus effectively suppresses pancreatic tumor growth and triggers synergistic anticancer effects with gemcitabine and nab-paclitaxel. Oncotarget. 2017;8(14):22700-22715.

24. Braiteh F, Patel MB, Parisi M, Ni Q, Park S, Faria C. Comparative effectiveness and resource utilization of nab-paclitaxel plus gemcitabine vs FOLFIRINOX or gemcitabine for the first-line treatment of metastatic pancreatic adenocarcinoma in a US community setting. Cancer Manag Res. 2017;9:141-148.

25. Goldstein D, Von Hoff DD, Moore M, et al. Development of peripheral neuropathy and its association with survival during treatment with nabpaclitaxel plus gemcitabine for patients with metastatic adenocarcinoma of the pancreas: a subset analysis from a randomised phase III trial (MPACT). Eur J Cancer. 2016;52:85-91.

26. De Vita F, Ventriglia J, Febbraro A, et al. NAB-paclitaxel and gemcitabine in metastatic pancreatic ductal adenocarcinoma (PDAC): from clinical trials to clinical practice. BMC Cancer. 2016;16(1):709.

27. Linee Guida AIOM Carcinoma del pancreas esocrino - Aiom 2017. Available from: http://www.aiom.it/professionisti/documenti-scientifici/ linee-guida/1,413,1. Accessed May 21, 2018.

28. Rajeshkumar NV, Yabuuchi S, Pai SG, et al. Superior therapeutic efficacy of nab-paclitaxel over cremophor-based paclitaxel in locally advanced and metastatic models of human pancreatic cancer. Br J Cancer. 2016; 115(4):442-453.

29. Zhou J, Zhao R, Wen F, et al. Cost-effectiveness analysis of treatments for metastatic pancreatic cancer based on PRODIGE and MPACT trials. Tumori. 2016;2016(3):294-300.

30. Chiorean EG, Von Hoff DD, Tabernero J, et al. Second-line therapy after nab-paclitaxel plus gemcitabine or after gemcitabine for patients with metastatic pancreatic cancer. Br J Cancer. 2016;115(2):188-194. 


\section{Publish your work in this journal}

Drug Design, Development and Therapy is an international, peerreviewed open-access journal that spans the spectrum of drug design and development through to clinical applications. Clinical outcomes, patient safety, and programs for the development and effective, safe, and sustained use of medicines are the features of the journal, which has also been accepted for indexing on PubMed Central. The manuscript management system is completely online and includes a very quick and fair peer-review system, which is all easy to use. Visit http://www.dovepress.com/testimonials.php to read real quotes from published authors.

Submit your manuscript here: http://www.dovepress.com/drug-design-development-and-therapy-journal 